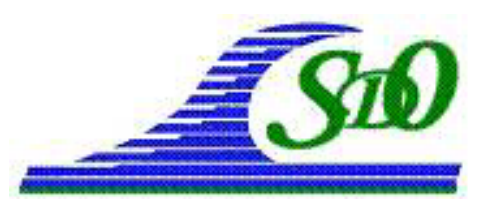

XI $^{\text {èmes }}$ Journées Nationales Génie Côtier - Génie Civil
Les Sables d'Olonne, 22-25 juin 2010

DOI:10.5150/jngcgc.2010.081-O @ Editions Paralia CFL

disponible en ligne - http://www.paralia.fr - available online

\title{
Simulation physique du frottement latéral à l'interface sol-pieu lors du fonçage (cas d'une interaction limon-acier)
}

\author{
Azzedine OULARBI ${ }^{1}$, Daniel LEVACHER ${ }^{2}$, Nabil MIMOUNI $^{3}$
}

1. Université Saad DAHLAB de Blida, Faculté des Sciences de l'Ingénieur, Département SEE, Route de soumaa, BP 270, 09000 Blida, Algérie.

Drazzoular@gmail.com

2. Université de Caen, Faculté des Sciences, UMR 6143 CNRS- M2C, Esplanade de la paix, 14032 Caen, France.

daniel.levacher@unicaen.fr

3. Ingénieur consultant, 15125 Westroad, Houston, TX, 77095 USA.

\section{Résumé :}

Les méthodes actuelles d'évaluation des charges de service par frottement latéral des pieux d'ancrages de fixation de structures fixes ou flottantes, utilisent des modèles simples adaptés uniquement aux conditions statiques; les caractéristiques du sol à l'interface sol-pieu sont prises en compte par des coefficients empiriques traduisant entre autre le frottement visqueux le long du fût du pieu.

Cependant, ces méthodes ne prennent pas en compte l'évolution des caractéristiques de l'interaction sol-pieu sous l'effet dynamique provoqué par la mise en place des pieux.

L'imprécision sur le comportement de l'interaction sol-pieu sur le fût est d'autant plus importante que l'incertitude des résultats fournis par ces méthodes de calcul est plus grande notamment dans le cas des sédiments très fins de type limoneux ou marneux (fonds marins).

Une nouvelle approche bien adaptée visant à simuler physiquement à $1 \mathrm{~g}$, à l'aide d'un dispositif expérimental instrumenté, le mécanisme de mobilisation du frottement latéral à l'interaction sol-pieu sous l'effet de fonçage, est adoptée dans cette étude expérimentale.

C'est l'analyse des résultats expérimentaux de la campagne d'essais ainsi réalisés qui est présentée dans cet article.

\section{Mots-clés :}

Fonçage - Pieux - Simulation physique - Frottement latéral - Limoneux - Interaction Mobilisation

\section{Introduction}

Le frottement représente la partie principale de la capacité axiale de pieux très long dit "flottant" servant d'appui à des structures fixes sur terre et offshore dans des eaux profondes. 
Dans ce cas, une estimation de la capacité portante est considérée comme proche de la réalité si elle tient compte des contraintes dues aux sollicitations dynamiques exercées lors de la mise en œuvre par un chargement dynamique tel que battage, vibropercussion ou vibrofonçage.

La prévision de cette capacité est d'autant meilleure que si, outre la prise en compte de l'effet de masse du sol et des caractéristiques géomécaniques de l'inclusion, le comportement de l'interface sol-inclusion est bien modélisée.

Dans cette optique divers travaux de recherche expérimentale et numérique ont été développés pour appréhender par différentes approches les mécanismes physiques de mobilisation $\mathrm{du}$ frottement à l'interface pendant un chargement dynamique (MIDDENDORP \& VAN BREDERODE, 1984 ; HEEREMA, 1979 ; LEVACHER, 1992).

La synthèse des résultats d'analyse de ces travaux à permis de montrer que le comportement réel, incluant la compressibilité du sol environnant, se situe entre l'essai de cisaillement direct, à contrainte normale constante, et l'essai à volume constant. L'influence de l'interface sol-inclusion est très importante mais difficilement quantifiable. Dans ce point de vue, cet article présente les principaux résultats d'une campagne d'essais effectués au laboratoire et réalisés à l'aide d'un dispositif expérimental spécialement conçu pour analyser les mécanismes de mobilisation du frottement latéral durant un chargement dynamique et ce dans le cas particulier d'une interaction limon-acier.

\section{Modèle d'expérimentation}

Devant la complexité de l'expérimentation sur des ouvrages en vraie grandeur en raison des moyens matériels énormes à mettre en œuvre, le modèle physique constitue une alternative très intéressante d'analyse des phénomènes physiques. Bien que le modèle présente certains inconvénients, tels que l'effet d'échelle, il n'en demeure pas moins qu'il est indispensable pour l'étude des ouvrages complexes tels que les pieux.

\subsection{Description du modèle et du dispositif expérimental}

Dans cette étude le frottement latéral lors de l'installation par un chargement dynamique est simulé à l'aide d'un modèle composé d'un support en forme de demi cylindre creux où loge l'échantillon de sol non remanié $(\varnothing=80 \mathrm{~mm}, \mathrm{~h}=160 \mathrm{~mm})$ et sur lequel il est appliqué une contrainte horizontale par l'intermédiaire d'un bras de levier supportant différentes masses.

Le déplacement vertical est généré à l'aide d'un vérin électrique à vitesse variable de 2 à $60 \mathrm{~mm} / \mathrm{s}$. L'extrémité du vérin est reliée à la plaque métallique à l'aide d'un embout à rotule afin d'éviter toute obliquité de la plaque lors de son déplacement.

L'acquisition des mesures est assurée par une chaîne équipée de capteur de force et d'un capteur de déplacement. Pour une meilleure optimisation de l'amplification et 
l'atténuation des bruits parasites le dispositif a été doté d'ampli-conditionneur et de système d'isolation. Le schéma détaillé du dispositif expérimental est représenté sur la figure 1.

\subsection{Le matériau d'essai (caractéristique physiques et mécaniques)}

Durant toute la campagne expérimentale, Il a été procédé à des essais sur des échantillons d'argile dont la granulométrie renferme 50 à $60 \%$ d'éléments inférieurs à $2 \mu$. La fraction fine est composée essentiellement de Montmorillonite, le reste en proportion plus faible d'Illite et de Kaolinite.

Les caractéristiques géotechniques des différents échantillons obtenus sur un sondage sont consignées dans le tableau 1.

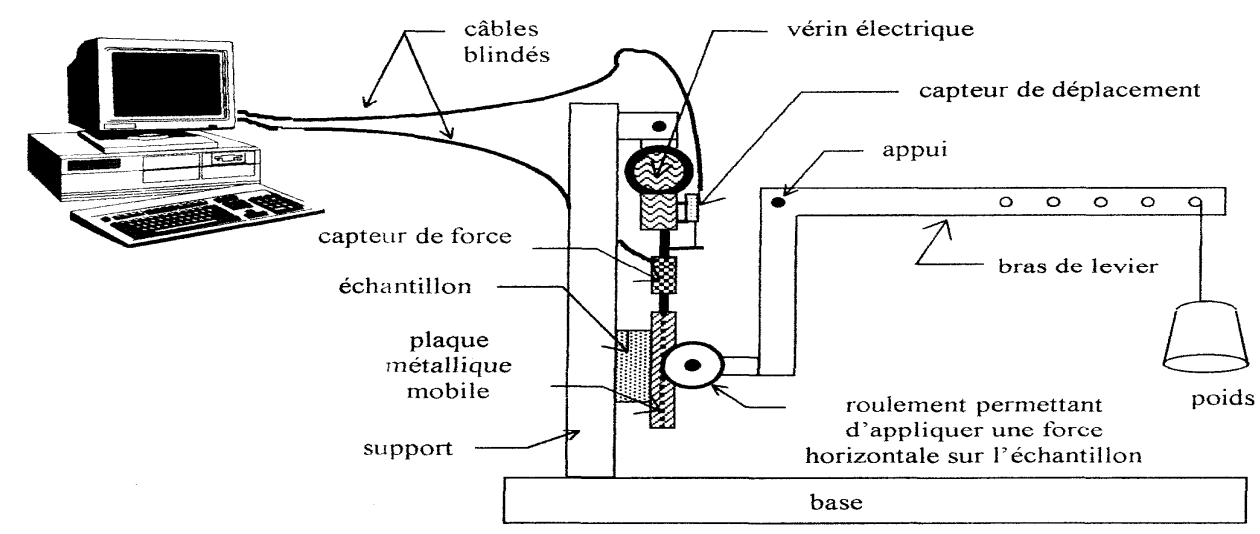

Figure 1. Dispositif expérimental de simulation physique de l'interaction sol-pieu (MIMOUNI, 1998).

Tableau 1. Caractéristiques des argiles testées.

\begin{tabular}{|c|c|c|c|c|c|c|c|}
\hline $\begin{array}{l}\text { Profondeur } \\
\text { (m) }\end{array}$ & $W(\%)$ & $W_{l}(\%)$ & $I_{p}$ & $\begin{array}{l}\gamma_{d} \\
\left(K N / m^{3}\right)\end{array}$ & $\begin{array}{l}\text { M.O. } \\
(\%)\end{array}$ & $\begin{array}{l}C_{u} \\
(K P a)\end{array}$ & $e$ \\
\hline $0 \grave{a} 2$ & 48 & 75 & 30 & 11. & 1 & 45 & 1,3 \\
\hline $2 \grave{a} 4$ & 108 & 125 & 82 & 6,6 & 3 & 15 & 2,9 \\
\hline $4 \grave{a} 8$ & 64 & 70 & 31 & 9,3 & 2 & 40 & 1,8 \\
\hline $8 \grave{a} 17$ & 78 & 87 & 47 & 9,1 & 4 & 39 & 1,7 \\
\hline
\end{tabular}

\section{Présentation des résultats expérimentaux}

La multitude des facteurs dans un phénomène rend difficile la réalisation d'un dispositif expérimental, pouvant tenir compte de tous les paramètres intervenant dans le phénomène. C'est pour cette raison que la campagne d'essais a été conduite en tenant en compte uniquement des paramètres suivants : la contrainte horizontale, la vitesse de 
déplacement de la plaque et les caractéristiques physique et mécanique des différents échantillons.

Pour tous les types d'essais ainsi réalisés (à contrainte horizontale constante et à vitesse constante), la mobilisation du frottement latéral est analysée à partir des courbes de variation du frottement latéral en fonction du déplacement.

Nous remarquons que l'allure des courbes obtenues sont assez typique et se caractérise généralement par deux phases; une phase de mobilisation quasi linéaire suivie d'une phase de rupture associée à un palier telle qu'illustré sur la figure 2 .

\subsection{Influence de la contrainte horizontale}

Afin de bien apprécier l'influence de la contrainte horizontale sur le frottement latéral à l'interface, on maintient la vitesse de déplacement de la plaque constante et on fait varier la contrainte horizontale jusqu'à une valeur limite fixée à $120 \mathrm{KPa}$. Il est à noter qu'au-delà de cette valeur limite, on observe un refoulement du sol plus ou moins accentué selon les caractéristiques géotechniques des échantillons utilisés.

Par ailleurs l'analyse des graphes frottement-déplacement révèle, durant la phase de mobilisation et pour les valeurs extrêmes de contrainte horizontale de 70 et $80 \mathrm{KPa}$ un caractère assez remarquable qui se traduit par la présence de deux pentes successives juste avant la phase de rupture comme le montre la figure 3.

A l'exception de ces valeurs extrêmes de la contrainte horizontale, le frottement semble se mobiliser suivant une seule pente jusqu'à la phase de rupture. On observe par ailleurs qu'une augmentation progressive de la contrainte horizontale entraîne à la fois un accroissement du frottement latéral durant la phase de mobilisation et la phase de la rupture comme le montre bien la figure 2 et la figure 4 .

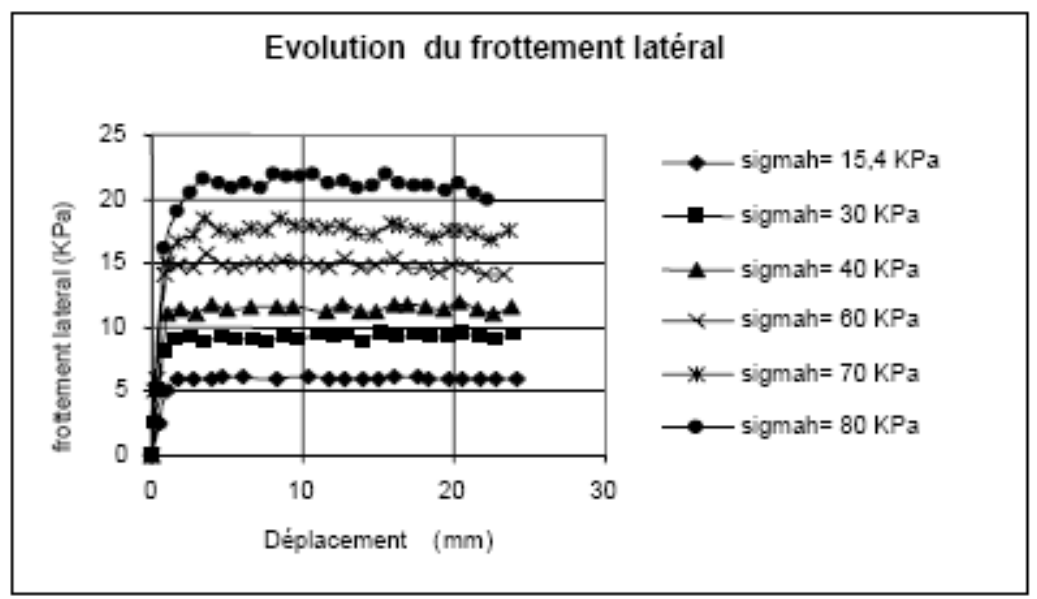

Figure 2. Evolution du frottement latéral à l'interface sol-plaque. 


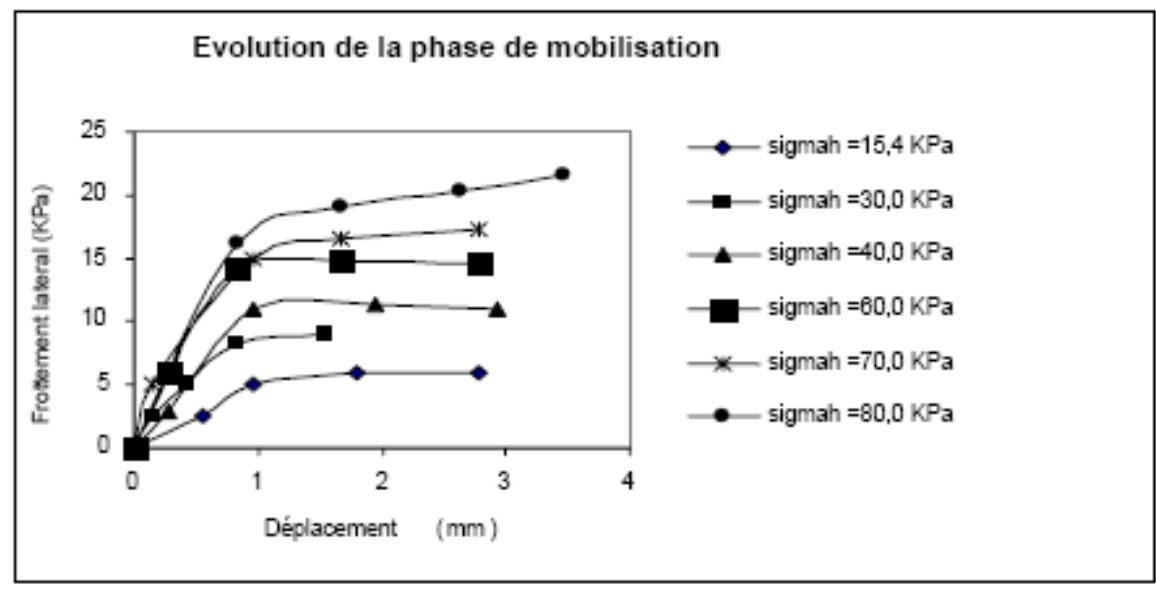

Figure 3. Caractérisation de l'effort latéral durant la phase de mobilisation.
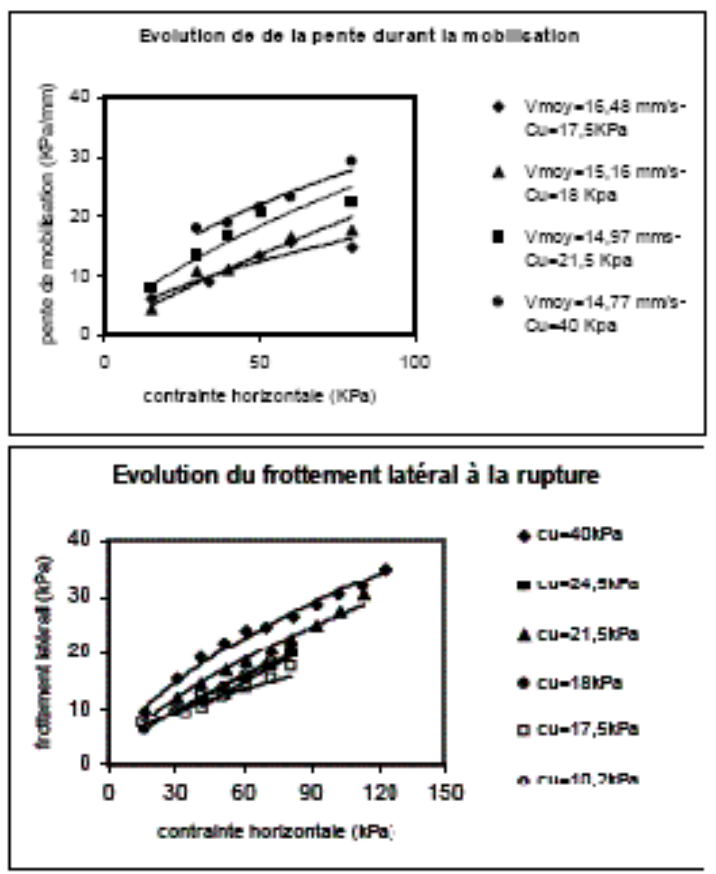

Figure 4. Variation de la pente de mobilisation et du frottement latéral à la rupture en fonction de la contrainte horizontale.

\subsection{Influence de la vitesse de déplacement}

La figure 5 présente l'influence de la vitesse de déplacement sur le frottement à l'interface. 


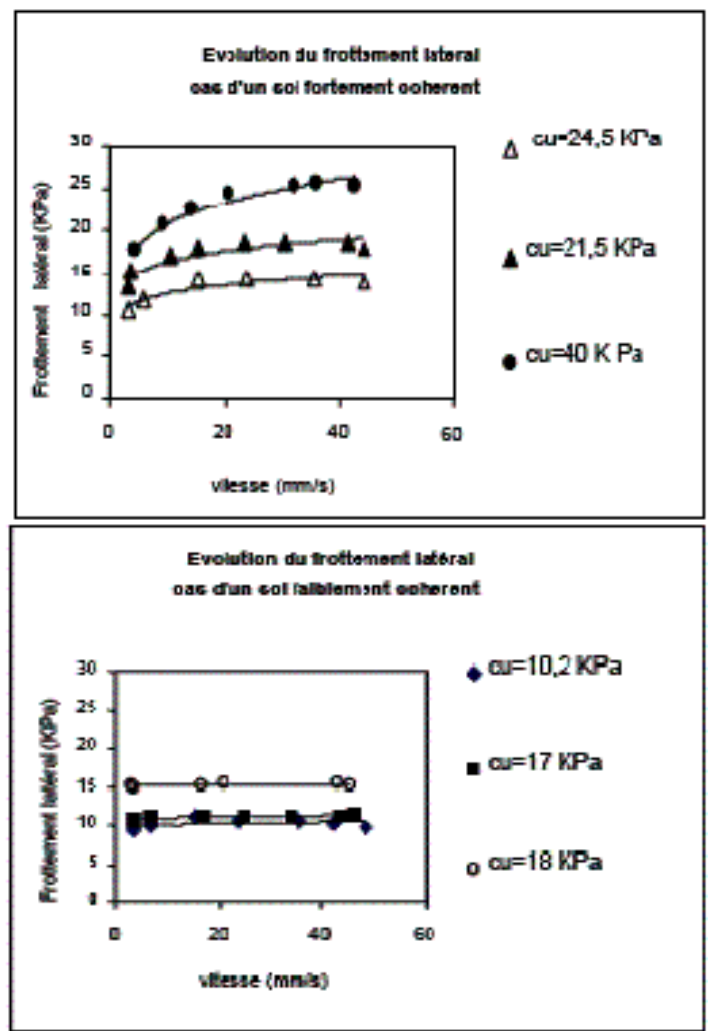

Figure 5. Variation du frottement à la rupture en fonction de la vitesse.

Les mêmes observations concernant la présence de double pente et de pente simple pendant la phase de mobilisation ont été relevées. Par contre, contrairement aux essais à vitesse constante, le frottement semble se mobiliser par une double pente pour des vitesses relativement faibles et par une seule pente pour des vitesses élevées et ceci indépendamment des caractéristiques physique et mécanique du sol.

En outre l'analyse des résultats de cette deuxième série a révélé que le frottement latéral à l'interface ne croit pas systématiquement avec la vitesse et semble dépendre de la cohésion.

L'influence de la teneur en eau reste dans le contexte de notre expérimentation assez ambiguë.

Bien que des similitudes dans les résultats des deux types d'essais aient été relevées il n'en demeure pas moins que l'influence de la cohésion du sol soit toutefois beaucoup plus marquée en particulier pendant la phase de rupture telle que représentée sur la figure 6. En effet nous notons un accroissement quasi linéaire du frottement avec la cohésion du sol et une augmentation régulière du frottement latéral à la rupture avec un accroissement progressive de la contrainte horizontale, ce qui réconforte le constat de l'analyse précédente. 


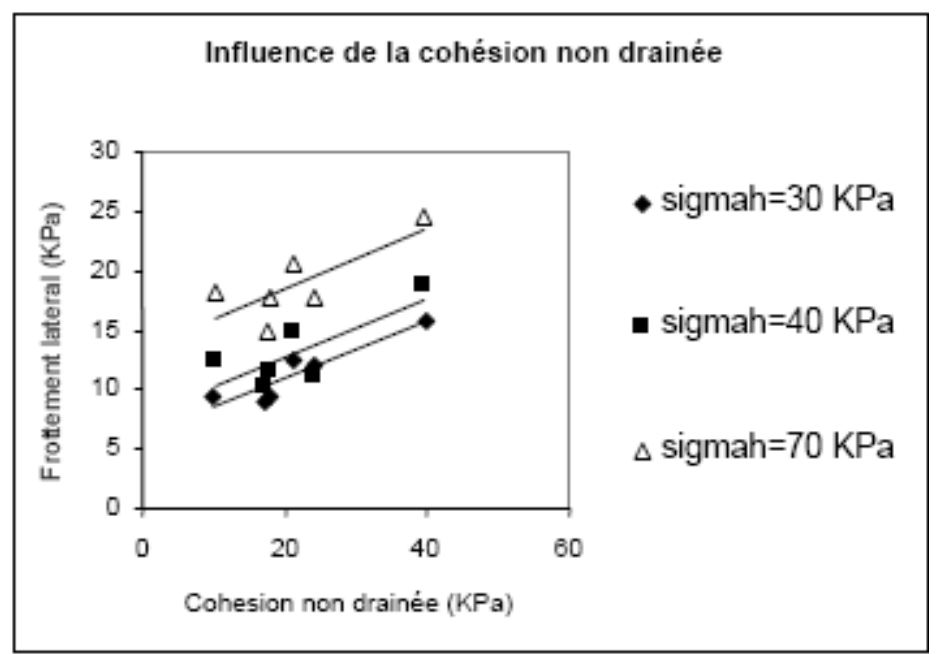

Figure 6. Variation du frottement à la rupture en fonction de la cohésion non drainée.

Nous notons également deux faits remarquables dans l'allure des courbes de variation $\mathrm{du}$ frottement à l'interface en fonction de la vitesse, depuis la mobilisation jusqu'à la rupture (figure 5).

Nous observons en particulier, pour les faibles valeurs de la cohésion non drainée, l'absence de l'influence de la vitesse sur l'évolution de la mobilisation du frottement latéral, a contrario, pour des valeurs relativement importantes de la cohésion non drainée, la mobilisation du frottement latéral semble augmenter rapidement sur l'intervalle des faibles valeurs de vitesse avant d'atteindre le seuil de la phase de rupture.

Ce phénomène, qui semble dépendre de la cohésion a été déjà été observé et interprété (HEEREMA 1979).

\section{Conclusion}

Les résultats de l'expérimentation menée sur modèle ont permis en premier lieu de valider une approche simplifiée d'interprétation du mécanisme de mobilisation du frottement à l'interface sol-structure, dans le cas particulier de sols cohérents, soumis a des sollicitation dynamiques.

Ces travaux ont permis de confirmer, d'une part, l'interprétation proposée par Heerema, d'autre part, de mettre en évidence le rôle important joué par la combinaison des différents facteurs tels que la contrainte horizontale, la vitesse, la cohésion et dans un degré moindre la teneur en eau.

Dans cet ordre d'idée, les bons résultats des recherches, une mise en œuvre facile et une bonne fiabilité de fonctionnement font du modèle un bon outil pour un apport dans la modélisation de l'interaction sol-pieu lors d'un chargement dynamique. 
Thème 5 -Ouvrages portuaires, côtiers et offshore

\section{Références bibliographiques}

HEEREMA E.P. (1979). Relationships between wall friction, displacement, velocity and horizontal stress in clay and in sand, for pile driveability analysis. Ground engineering, pp 55-65.

MIDDENDORP P., VAN BREDERODE P.J. (1984). Skin friction models for sand from static and dynamic laboratory load tests. Proc. $2^{\text {nd }}$ international conference on the application of stress wave theory on piles, Stockholm, pp 210-220.

LEVACHER D. (1992). Modélisation numérique et physique du battage des pieux. Simulation en centrifugeuse du comportement des pieux. Thèse de Doctorat d'Etat, Ecole Centrale de Nantes.

MIMOUNI N. (1998). Etude expérimentale du frottement latéral a l'interface solstructure en dynamique: cas d'une interaction limon-acier. Thèse de Doctorat, université du Havre. 